\title{
The need for the fractional operators
}

\author{
E. A. Abdel-Rehim*
}

${ }^{*}$ Correspondence:

entsarabdelrehim@yahoo.

com

Department of Mathematics,

Faculty of Science, Suez

Canal University, Ismailia,

Egypt

\begin{abstract}
In this review paper, I focus on presenting the reasons of extending the partial differential equations to space-time fractional differential equations. I believe that extending any partial differential equations or any system of equations to fractional systems without giving concrete reasons has no sense. The experiments agrees with the theoretical studies on extending the first order-time derivative to time-fractional derivative. The simulations of some processes also agrees with the theory of continuous time random walks for extending the second-order space fractional derivative to the Riesz-Feller fractional operators. For this aim, I give a condense review the theory of Brownian motion, Langevin equations, diffusion processes and the continuous time random walk. Some partial differential equations that are successfully extended to space-timefractional differential equations are also presented.
\end{abstract}

Keywords: Space-fractional Fokker-Planck operator, Caputo-time-fractional operator, Riesz-Feller space-fractional operator Mittag-Leffler function, Continuous time random walk

Mathematics Subject Classification: 26A33, 35L05, 35J05, 45K05, 60J60, 60G50, 60G51, 65N06, 80-99, 42A38, 33C20, 44A10

\section{The need for time-fractional differential operators}

The random motion, the erratic motion, of a small particle immersed in a fluid is called the Brownian motion and is mathematically modelled by the diffusion equation

$$
\frac{\partial u(x, t)}{\partial t}=D \frac{\partial^{2} u(x, t)}{\partial x^{2}}, u(x, 0)=\delta\left(x-x_{0}\right),
$$

where its solution is

$$
u(x, t)=\frac{1}{2 \sqrt{\pi D t}} \mathrm{e}^{-\left(x-x_{0}\right)^{2} /(4 D t)},
$$

which is typical Gaussian distribution. Albert Einstein deduced the value of the diffusion constant $D$ as

$$
D=\frac{R T}{6 N_{A} \pi \eta r}=\frac{k_{\mathrm{B}} T}{6 \pi \eta r},
$$
author(s) and the source, provide a link to the Creative Commons licence, and indicate if changes were made. The images or other third party material in this article are included in the article's Creative Commons licence, unless indicated otherwise in a credit line to the material. If material is not included in the article's Creative Commons licence and your intended use is not permitted by statutory regulation or exceeds the permitted use, you will need to obtain permission directly from the copyright holder. To view a copy of this licence, visit http:// creativecommons.org/licenses/by/4.0/. 
where $R$ is the gas constant, $N_{\mathrm{A}}$ is the Avogadro number, $T$ is the temperature, $\eta$ is the liquid's velocity, and $r$ is the radius of the Brownian particle. Let $m$ be the mass of the Brownian particle, see $[1,2]$, the Newton's equation of motion reads

$$
m \frac{\mathrm{d} v(t)}{\mathrm{d} t}=F(t)
$$

where $F(t)$ is the instantaneous force of the particle at time $t$. Let $F(t)=-\gamma v(t)$, i.e. it represents the friction force. By Stoke's law, the friction coefficient is $\gamma=6 \pi \eta r$. Now let $\zeta(t)$ be the random force of the random fluctuation of the fluid, then (1.2), takes the form

$$
\frac{\mathrm{d} v(t)}{\mathrm{d} t}=-\frac{\gamma}{m} v(t)+\frac{\zeta(t)}{m} .
$$

This equation is called the Langevin equation of motion. Here $\zeta(t)$, is a stochastic variable representing the noise due to the fluid on the Brownian erratic particle. Neglecting the effect of $\zeta(t)$ and solve Eq. (1.3), to get

$$
v(t)=v(0) \mathrm{e}^{-\frac{t}{\tau_{B}}}, \tau_{B}=\frac{m}{\gamma}
$$

where $v(0)$ is the initial velocity of the suspended erratic particle. If $\tau_{B}>>t$, then $v(t)=1-\frac{t}{\tau_{B}}$. Integrating (1.4) with respect to $t$, one gets

$$
x(t)=x_{0}+\int_{0}^{t} v(s) \mathrm{d} s
$$

, and consequently, the average displacement reads

$$
<x(t)>=x_{0}+v(0) \tau_{B}\left(1-\mathrm{e}^{\left(-\frac{t}{\tau_{B}}\right)}\right) .
$$

That means the Langevin equation (1.3) with zero noise is equivalent to the diffusion equation (1.1) under the natural assumptions

$$
u(x, t) \rightarrow 0, x^{n} u(x, t) \rightarrow 0 \text { as }|x| \rightarrow \infty \text { and } \int_{-\infty}^{\infty} u(x, t) \mathrm{d} x=1 .
$$

If the noise at the Langevin equation (1.3) is not neglected, and by taking into consideration the same natural assumptions, Eq. (1.3) turns to the Fokker-Plank equation (the forward Kolmogorov equation ), namely

$$
\frac{\partial u(x, t)}{\partial t}=\frac{1}{2} \frac{\partial^{2} a(x, t) u(x, t)}{\partial x^{2}}-\frac{\partial b(x, t) u(x, t)}{\partial x}=L_{f p} u(x, t), u(x, 0)=\delta(x) .
$$

Suppose that the functions $a(x, t)$ and $b(x, t)$ are power functions of $x$ only or constants, to be able to use the natural properties, namely (1.6). The first moment of equation (1.7) is gotten by multiply each sides of Eq. (1.7) by $x$ and integrate over $x \in \mathbb{R}$, to get the initial value problem 


$$
\frac{\mathrm{d}\langle x(t)\rangle}{\mathrm{d} t}=-\langle x(t)\rangle
$$

whose solution is

$$
m(t)=\langle x(t)\rangle \approx\left\langle x_{0}\right\rangle \mathrm{e}^{-t} .
$$

This equation is considered as a Markov process with exponential waiting time. For more details about the theory of the Brownian motion, the passage limit to diffusion processes and the Fokker Planck equations, see [3-11]. The partial differential equations of type (1.7) and their solutions have the previous natural properties that covers a huge number of applications on biology, physics, chemistry, medicine and engineering.

So far, I have reviewed the relation between the stochastic Langevin equation and the classical Diffusion process. Not only the diffusion equations are associated with the FokkerPlanck operator and its special cases but also the movement of the potential and current in an electric transmission line (Cable equation), namely

$$
\frac{\partial^{2} I(x, t)}{\partial t^{2}}+k c^{2} \frac{\partial I(x, t)}{\partial t}=c^{2} \frac{\partial^{2} I(x, t)}{\partial x^{2}}+b c^{2} I(x, t)=L_{f p} I(x, t),
$$

with the resistance $R$, inductance $L$, capacitance $c$ and leakage conductance $G$, the resistance $k$, and the function $I(x, t)$ to represent the electric transmitted current. If $I(x, t) \rightarrow 0$ and $x^{n} I(x, t) \rightarrow 0$ as $|x| \rightarrow \infty$, then it could be specified as stochastic processes, see [14].

Consider the natural generalisation of the first-order-time derivative, namely the Caputotime-fractional operator that reads

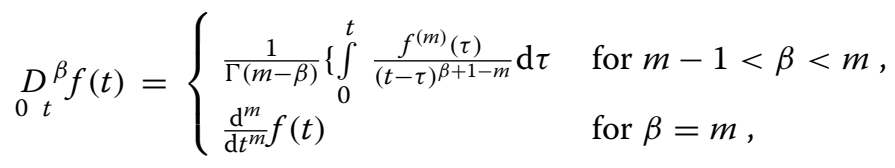

where

$$
K_{\beta}(t-\tau)=\frac{(t-\tau)^{\beta+1-m}}{\Gamma(m-\beta)},
$$

is its kernel and is called the memory function. This kernel enables reflects the memory effects of many physical, biological, etc., processes. The Caputo-time fractional derivative $D_{0}^{\beta}$ can also be defined through its image in the Laplace transform domain, which is

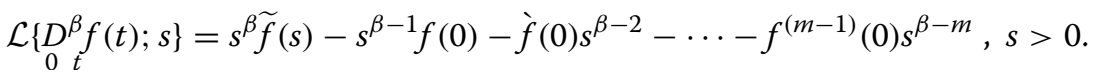

Now, rewrite the classical diffusion equation (1.7) as

$$
{\underset{0}{D}}_{t}^{\beta} u(x, t)=L_{f p} u(x, t), u\left(x, x_{0}\right)=\delta\left(x-x_{0}\right), 0<\beta \leq 1 .
$$

This equation could be written as

$$
u(x, t)-u(x, 0)=D_{t}^{-\beta}\left(L_{f p} u(x, t)\right)
$$


where $D_{t}^{-\beta}=J^{\beta}$ is the Riemman-Liouville fractional integral operator, see [15-17]. The first moment of this time fractional diffusion equation satisfies

$$
D_{t}^{D^{\beta}}\langle x(t)\rangle=-\langle x(t)\rangle
$$

, and its solution is

$$
m(t)=\langle x(t)\rangle \approx\left\langle x_{0}\right\rangle E_{\beta}\left(-t^{\beta}\right),
$$

where $E_{\beta}\left(-t^{\beta}\right)$ is the Mittag-Leffler function of order $\beta$, see [12] for the basic properties of the Mittag-Leffler function. The general form of the infinite series of the Mittag-Leffler function of order $\alpha$ and $\beta$ is defined as

$$
E_{\alpha, \beta}(z)=\sum_{k=0}^{\infty} \frac{z^{k}}{\Gamma(\beta+k \alpha)}, \alpha \in \mathbb{R}, \beta \in \mathbb{R}, z \in \mathbb{C},
$$

where $E_{0,1}(z)=\mathrm{e}^{z}$. Gorenflo et. al. [13] introduced the very important role of Mittag-Leffler function and its asymptotic behaviours on simulating the continuous time random walks. Therefore, the processes that their velocities and first moments behave asymptotically as $t^{-\beta}$ and $0<\beta<1$, and their solutions have the same stated natural properties (1.6) that can be extended to time-fractional differential equations, see also [17]. Even the time-fractional Cable equation with time-fractional damping term that reads

$$
{\underset{0}{D}}_{t}^{\beta} I(x, t)+\underset{0}{k D_{t}^{\gamma}} I(x, t)=L_{f p} u(x, t) 0<\beta<2,0<\gamma<1
$$

is a natural generalisation of its classical version (1.8) only if its solution $I(x, t)$ satisfies the condition (1.6), and its average is

$$
m(t)=\langle x(t)\rangle=\left\langle x_{0}\right\rangle \frac{m^{n}}{n !} t^{-n \beta+\beta-\gamma} E_{(1+\beta-\alpha),(1+n \beta)}^{(n)}\left(-k t^{(\beta-\alpha)}\right),
$$

where $E_{(1+\beta-\gamma),(1+n \beta)}^{(n)}\left(-k t^{(\beta-\gamma)}\right)$ is the $n$th derivative of $E_{(1+\beta-\alpha),(1+n \beta)}\left(-k t^{(\beta-\alpha)}\right)$. That means its velocity variations are also asymptotically behaves as $t^{-(\beta-\alpha)}$, see [18]. The processes that are satisfied that the previous properties can be extended to time-fractional differential equation. In some cases as the genetic drift process of asexual population, that is mathematically modelled by the following equation

$$
\begin{aligned}
\frac{\partial u(p, x ; t)}{\partial t}= & \frac{1}{N} \frac{\partial^{2}}{\partial x^{2}}\{x(1-x) u(p, x ; t)\} \\
& +\left(\mu_{+}-\mu_{-}\right)[1-\zeta(1-s)] \frac{\partial}{\partial x}\{x(1-x) u(p, x ; t)\} \\
& -N \zeta s\left[x \mu_{+}+(1-x) \mu_{-}\right] u(p, x ; t),
\end{aligned}
$$

where $N$ is the total population size, $\mu_{+}$is the mutator mutation rate per genome, $\mu_{-}$is the wild-type mutation rate per genome, $\zeta$ is the fraction of beneficial mutations, and $s$ is the selection coefficient of mutation, one cannot extend its first time derivative to the Caputo time-fractional derivative. Abdel-Rehim et. al. [19] proved that although this process satisfies the natural conditions (1.6), imposed on its solution but as asexual self 
proliferation, the parent inherits all its genes and characteristics to his offspring. Also the simulation shows that the effects on the memory are neglect because only the wildtype mutator plays the significant rule on this asexual diffusion process

\section{The need for space-fractional differential operators}

One needs the space-fractional operators to mathematically model many physical, biological, chemical, medical, etc., phenomena that move through fractal media and usually exhibit large deviations from the Brownian motion (1.1) and do not require finite velocity. The extension to Lévy stable [20] motion is a straight forward generalisation, namely

$$
\frac{\partial u(x, t)}{\partial t}=\underset{0 x, \theta}{D}{ }^{\alpha} u(x, t), u(x, 0)=f(x)=\delta\left(x-x_{0}\right), 0<\alpha \leq 2 .
$$

${ }_{0}{ }_{x, \theta}^{\alpha}$ is called the Riesz-Feller fractional operator. Gorenflo and Mainardi [21], proved that the Riesz-Feller operator as, $\theta=0$, is formally a power of the positive definitive operator $\underset{0 x}{D_{x}^{2}}=-\frac{\mathrm{d}^{2}}{\mathrm{~d} x^{2}}$, i.e. $-\left(\underset{0}{-D^{2}}\right)^{\alpha / 2}=\underset{0}{D_{x}^{\alpha}}$, i.e. the generalisation to the fractional differential operator is natural. The Fourier transform of (2.1) reads

$$
\frac{\partial u(\hat{\kappa}, t)}{\partial t}=-D|\kappa|^{\alpha} \mathrm{e}^{i \theta \pi} \operatorname{sig}(\kappa) u(\hat{\kappa}, t)
$$

where $\theta$ is the parameter of asymmetry (skewness) which is introduced by Feller, see Feller [5], Gorenflo et. al. [21, 22]. These pioneer researchers proved that the characteristic function $\widehat{u}(\kappa, t)$ belongs to the class of $\alpha$-stable probability densities that reads

$$
\widehat{g}_{\alpha}(\kappa, t ; \theta)=\exp \left[-t|\kappa|^{\alpha} \mathrm{e}^{\frac{i \theta \pi}{2} \operatorname{sig}(\kappa)}\right], \widehat{u}(\kappa, 0)=\widehat{p}(\kappa),
$$

where $\widehat{g}_{\alpha}(\kappa, t ; \theta)$ is the Fourier transform of the Green function corresponding to the initial condition $g_{\alpha}(x, 0 ; \theta)=\delta(x)$. The solution of Eq. (2.1) can be written as

$$
u(x, t)=\int_{-\infty}^{\infty} g_{\alpha}(x-\zeta, t ; \theta) f(\zeta) \mathrm{d} \zeta, \forall t>0
$$

and the special case as $\theta=0$, one has

$$
u(\hat{\kappa}, t)=\mathrm{e}^{-D t|\kappa|^{\alpha}} .
$$

The asymptotic behaviour of this solution is

$$
u(x, t) \approx \frac{D t}{|x|^{1+\alpha}},
$$

, and this is called the Lévy long tail. This agrees with the theory of stochastic processes having $\alpha$-stable distribution. In which the jump density function for the random jump $X$ is denoted by $p(x)$, and its characteristic function $\hat{p}(\kappa)$, see [23], is defined as

$$
\hat{p}(\kappa)=\int_{-\infty}^{\infty} \mathrm{e}^{i \kappa x} p(x) \mathrm{d} x .
$$


It is known that the Feller-Riesz operator is symmetric as $p(x)=1-p(-x) \hat{p}(-\kappa)=\hat{p}(\kappa)$. Gendenko and Kolmogorov [24] proved that the jump width density function of Feller-Riesz operator belongs to the $\alpha$-stable distribution of the form

$$
L_{\alpha}=\log \widehat{p}(\kappa)= \begin{cases}i \mu^{\prime} \kappa-c|\kappa|^{\alpha}\left\{1+i \beta^{\prime} \frac{\kappa}{|\kappa|} w(|\kappa|, \alpha)\right\} & \text { if } \alpha \neq 1 \\ i \mu^{\prime} \kappa-c|\kappa|\left\{1+i \beta^{\prime} \frac{\kappa}{|\kappa|} w(|\kappa|, \alpha)\right\} & \text { if } \alpha=1\end{cases}
$$

where $\kappa \in \mathbb{R}, c \geq 0, \mu^{\prime}>0$ and $\left|\beta^{\prime}\right| \leq 1$. $\beta^{\prime}$ is the symmetry parameter. It determines the skewness of the distribution. $\beta^{\prime}=0$ is corresponding to a symmetric distribution. $c$ is the scale parameter. It determines the spread of the samples from a distribution around the mean. $\mu^{\prime}$ is the location parameter, and $\operatorname{Exp}\left[i \mu^{\prime} \kappa\right]$ basically corresponds to the shift in the $x$-axis of the probability density function. For $1<\alpha \leq 2, \mu^{\prime}$ represents the mean and for $0<\alpha \leq 1$, it represents the median. The stable distribution is said to be standard if $\mu^{\prime}=0$ and $c=1$. The function $w(\kappa, \alpha)$ is defined as

$$
w(|\kappa|, \alpha)= \begin{cases}\tan \frac{\pi \alpha}{2} & \text { if } \alpha \neq 1 \\ (2 / \pi) \log |\kappa| & \text { if } \alpha=1, \in \mathbb{R} .\end{cases}
$$

Abdel-Rehim [25] studied the fundamental solutions of the space-fractional diffusion and that are driven by using the Lévy convergent distribution functions $L_{\alpha}$. One can notes that the regular Brownian motion differs than the Lévy flights by the occurrence of extremely long jumps $\sim|x|^{-1-\alpha}, 0<\alpha<2$. Abdel-Rehim $[17,26]$ studied the theory of the continuous random walk and its relation to the space-fractional operators. AbdelRehim [17] and [26] presented the simulation of the continuous time random walk of the space fractional differential equation (2.1) and equation (1.7) after replacing $L_{f p}$ by $L_{f p}^{\alpha}$ that reads

$$
L_{f p}^{\alpha}=\underset{0 x}{D_{x}^{\alpha}}(a(x, t) u(x, t))-\frac{\partial b(x, t) u(x, t)}{\partial x},
$$

for all values of $\alpha$ and $\beta$. The simulations are consistent with the theory of the Brownian motion i.e. as $\alpha=2$ and the Lévy distribution as $0<\alpha<1, \alpha=1$ and $1<\alpha<2$. Sofar, the processes that their random walks exhibit Lévy flights and satisfy that the natural properties (1.6) could be mathematically modelled by space-fractional differential equations. Theses processes are always related to the diffusion of particles, solutes or gases in fractal medias.

Finally, Not any partial differential equation should be extended to space-fractional differential equation.

Authors' contributions

I Design, coordinate, write, and I have the idea of this manuscript as the single author.

Funding

There is no source of funding.

Availability of data and materials

Please contact author for data requests. 


\section{Declarations}

Competing interests

I declare that I have no competing interests.

Received: 19 August 2021 Accepted: 4 December 2021

Published online: 24 December 2021

\section{References}

1. Wang, M.C., Uhlenbeck, G.E.: On the theory of Brownian motion П. Rev. Mod. Phys. 17, 323-342 (1956)

2. Bharucha-Reid, A.T.: Elements of the Theory of Markov Processes and Their Applications. McGRAW-Hill book Company, New York (1960)

3. Smoluchowski, Drei Vortrage über Diffusion, Brownsche Molekularbewegung und Koagulation von Kolloidteilchen, Physikalische Z., 17, 557-571 (1916)

4. Uhlenbeck, G.E., Ornstein, L.S.: On the theory of Brownian motion. Phys. Rev. 36, 823-841 (1930)

5. Feller, W:: An Introduction to Probability Theory and Its Applications, vol. 1, 3rd edn. London, Sydney, New York (1968)

6. Kac, M.: Random walk and the theory of Brownian motion. In: The Annual Meeting of the Association at Swarthmore, New York (1946)

7. Ross Sheldon, M.: Introduction to Probability and Statistics for Engineering and Scientists, New York, Chichester. Toronto, and Singapore, Brisbane (1987)

8. Risken, H.: The Fokker-Planck Equation (Methods of Solution and Applications), 2nd edn. Springer, Berlin (1989)

9. Prabhu, N. U.: Stochastic Processes (Basic Theory and Its Applications). The Macmillan Company New York, CollierMacmillan Limited London (1965)

10. Kampan, N.G.V.: Stochastic Processes in Physics and Chemistry. North-Holland Publishing Company, Amsterdam (1981)

11. Vincze, l.: Über das Ehrenfestsche Modell der Wärmeüertragung. Arch. Math. 15, 394-400 (1964)

12. Erdélyi, A., Magnus, W., Oberhettinger, F., and Tricomi, F. G.: Higher Transcendental Functions, Bateman Project, Vols. 1-3, McGraw-Hill, New York (1953-1955)

13. Gorenflo, R., Abdel-Rehim, E.A.: Simulation of continuous time random walk of the space-fractional diffusion equations. J. Comput. Appl. Math. 222, 274-283 (2008)

14. Schneider, W.R., Wyss, W.: Fractional diffusion and wave equations. J. Math. Phys. 30(1), 134-144 (1989)

15. Samko, S.G., Kilbas, A.A., and Marichev, O.I.: Fractional Integrals and Derivatives(Theory and Applications). OPA, Amsterdam (1993)

16. Nonnenmacher, T.F., Metzler, R.: On the Riemann-Liouville fractional calculus and some recent applications. Fractals 3(3), 557-566 (1995)

17. Abdel-Rehim, E.A.: From the space-time fractional integral of the continuous time random walk to the space-time fractional diffusion equations, a short proof and simualtion. Physica A Stat. Mech. Appl. 531, 121547-121557 (2019). https://doi.org/10.1016/j.physa.2019.121547

18. Abdel-Rehim, E.A.: The approximate and the analytic solutions of the time-fractional intermediate diffusion wave equation associated with Fokker-Planck operator and applications. Math. Anal. Appl. (submitted)

19. Abdel-Rehim, E.A., Hassan, R.M., El-Sayed, A.M.A.: Markov and Non-Markov hereditary processes in asexual and random mating sexual populations, 12(3) No. 2 (2021) 1-15. 1st Inter. E-Conf.nin Math. Sciences and Fractional Calculus (ICMSFC Feb 2021). http://math-frac.org/Journals/JFCA

20. Lévy, P.: Théorie de l'addition des variables aleátoires. Gauthiers-Villars, Paris (1937)

21. Gorenflo, R., Mainardi, F.: Random walk models for space-fractional diffusion processes. Fract. Cal. Appl. Anal. 1, 167-191 (1998)

22. Mainardi, F., Pagnini, G., and Gorenflo, R.: Probability distributions as solitions to fractional diffusion equations. In: O.E. Barndorff-Nielsen (ed.), Mini-Proceedings: 2nd MaPhySto Conference on Lévy Processes: Theory and Applications, Dept. Mathematics, University of Aarhus, Denmark, 21-25 January 2002 (ISSN 1398-5957), pp. 197-205, 2002. [Available c/o http://www.MaPhySto.dk, Miscellanea No. 22]

23. Lukacs, E.: Charcteristic Functions. Charles Griffin and Company Limited, London (1960)

24. Gnedenko, B.V., Kolmogorov, A.N.: Limit Distributions for Sums of Independent Random Variables, (transl. from Russian by K. I. Chung). Addison-Wesley Publishing Company, Cambridge (1954)

25. Abdel-Rehim, E.A.: Fundamental solutions of the fractional diffusion and the fractional Fokker-Planck equations. J. Egypt. Math. Soc. 24, 337-347 (2016)

26. Abdel-Rehim, E.A.: From Power Laws to Fractional Diffusion Processes with and without External Forces, the Non Direct Way, Fractional Calculus and Applied Analysis, V. 22 No. 1 (2019) 60-77. This paper won the "Rodulf Gorenflo Award for young Researchers in Fractional Calculus" at the Conference ICFDA 2018

\section{Publisher's Note}

Springer Nature remains neutral with regard to jurisdictional claims in published maps and institutional affiliations. 